\title{
The XMM-Newton/INTEGRAL monitoring campaign of IGR J16318-4848
}

\author{
A. Ibarra ${ }^{1}$, G. Matt ${ }^{2}$, M. Guainazzi ${ }^{3}$, E. Kuulkers ${ }^{4}$, E. Jiménez-Bailón ${ }^{2}$, J. Rodriguez $^{5}$, F. Nicastro ${ }^{6}$, and R. Walter ${ }^{7}$ \\ 1 XMM-Newton Science Operations Center, European Space Astronomy Center, INSA, Apartado 50727, 28080 Madrid, Spain \\ e-mail: Aitor.Ibarra@sciops.esa.int \\ 2 Universitá degli Studi "Roma Tre", Via della Vasca Navale 84, 0046, Roma, Italy \\ 3 XMM-Newton Science Operations Center, European Space Astronomy Center, ESA, Apartado 50727, 28080 Madrid, Spain \\ ${ }^{4}$ Integral Science Operations Center, European Space Astronomy Center, ESA, Apartado 50727, 28080 Madrid, Spain \\ 5 AIM - Unité Mixte de Recherche CEA - CNRS - Université Paris VII - UMR 7158, CEA Saclay, Service d'Astrophysique, \\ 91191 Gif-sur-Yvette, France \\ 6 Harvard-Smithsonian Center for Astrophysics, Cambridge, MA, 01238, USA \\ 7 Integral Science Data Centre, Chemin d'Ecogia 16, 1290 Versoix, Switzerland
}

Received 10 August 2006 / Accepted 27 October 2006

\section{ABSTRACT}

Context. IGR J16318-4848 is the prototype and one of the more extreme examples of the new class of highly obscured Galactic X-ray sources discovered by INTEGRAL. A monitoring campaign on this source has been carried out using XMM-Newton and INTEGRAL, consisting of three simultaneous observations performed in February, March and August 2004.

Aims. The long-term variability of the Compton-thick absorption and emission line complexes will be used to probe the properties of the circumstellar matter.

Methods. A detailed timing and spectral analysis of the three observations is performed, along with the reanalysis of the XMM-Newton observation performed in February 2003. The results are compared with predictions from numerical radiative transfer simulations to derive the parameters of the circumstellar matter.

Results. Despite the large flux dynamic range observed (almost a factor 3 between observations performed a few months apart), the source remained bright (suggesting it is a persistent source) and Compton-thick $\left(N_{\mathrm{H}}>1.2 \times 10^{24} \mathrm{~cm}^{-2}\right)$. Large Equivalent Width (EW) emission lines from $\mathrm{Fe} \mathrm{K}_{\alpha}, \mathrm{Fe} \mathrm{K}_{\beta}$ and $\mathrm{Ni} \mathrm{K}_{\alpha}$ were present in all spectra. The addition of a $\mathrm{Fe} \mathrm{K}_{\alpha}$ Compton Shoulder improves the fits, especially in the 2004 observations. Sporadic occurrences of rapid X-ray flux risings were observed in three of the four observations. The $\mathrm{Fe} \mathrm{K}_{\alpha}$ light curve followed the continuum almost instantaneously, suggesting that the emission lines are produced by illumination of small-scale optically-thick matter around the high-energy continuum source. Using the iron line EW and Compton Shoulder as diagnostic of the geometry of the matter, we suggest that the obscuring matter is in a flattened configuration seen almost edge-on.

Key words. X-rays: individuals: IGR J16318-4848 - X-rays: binaries - line: formation - accretion, accretion disks

\section{Introduction}

IGR J16318-4848 was discovered by INTEGRAL on January 29th 2003, by the IBIS/ISGRI instrument, during a routine Galactic Plane Scan (Courvoisier et al. 2003). Re-analysis of archival ASCA data revealed at that position a highly absorbed $\left(N_{\mathrm{H}} \sim 10^{24} \mathrm{~cm}^{-2}\right)$ source (Murakami et al. 2003; Revnivtsev et al. 2003).

The discovery of this source triggered a XMM-Newton Target of Opportunity observation on February 10, 2003, and the subsequent analysis of the XMM-Newton data, in the $5-15 \mathrm{keV}$ energy band, confirmed the presence of a heavily absorbed source, $N_{\mathrm{H}} \sim 2 \times 10^{24} \mathrm{~cm}^{-2}$, with strong emission lines (Schartel et al. 2003).

This complex emission could be resolved in three components, with centroid energies of $6.410 \pm 0.003 \mathrm{keV}, 7.09 \pm$ $0.02 \mathrm{keV}$ and $7.47 \pm 0.02 \mathrm{keV}$, that correspond to $\mathrm{Fe} \mathrm{K}_{\alpha}, \mathrm{Fe} \mathrm{K}_{\beta}$, and $\mathrm{Ni} \mathrm{K}_{\alpha}$ fluorescent emission lines respectively (de Plaa et al. 2003). These preliminary results also showed that, during the XMM-Newton observation, IGR J16318-4848 was obscured by a Compton-thick absorber, $N_{\mathrm{H}}=(1.66 \pm 0.16) \times 10^{24} \mathrm{~cm}^{-2}$. A detailed analysis of this observation can be found in Matt \& Guainazzi (2003) and Walter et al. (2003).
Further studies at different wavelengths have also been carried out on the source. Filliatre \& Chaty (2004) found the optical counterpart of IGR J16318-4848. Their studies suggest that the source is a High Mass X-ray Binary (HMXB) at a distance between 1 and $6 \mathrm{kpc}$, the mass donor being an early-type star, probably a $s g B[e]$ star, surrounded by dense and absorbing circumstellar material. The $s g B[e]$ stars are massive, evolved, highluminosity stars undergoing mass loss in a two-component wind (see, e.g., Hynes et al. 2002).

The prominent, large equivalent width $(\mathrm{EW})$ of the $\mathrm{Fe} \mathrm{K}_{\alpha}$, $\mathrm{Fe} \mathrm{K}_{\beta}$, and $\mathrm{Ni} \mathrm{K}_{\alpha}$ fluorescent emission lines, observed in the XMM-Newton spectrum of IGR J16318-4848, makes this object one of the most extreme examples of the new class of highly absorbed X-ray binaries discovered by INTEGRAL (see, e.g., Kuulkers 2005 for a review).

A XMM-Newton observation campaign was carried out during 2004. At the same time, INTEGRAL was also observing the source, collecting data at energies higher than $10 \mathrm{keV}$. Due to its prominent, large $\mathrm{EW} \mathrm{Fe}$ and $\mathrm{Ni}$ emission lines, IGR J16318-4848 is an almost unique laboratory to study the physical condition and geometrical distribution of matter accreting on the compact object in a binary system. Due to this monitoring campaign we are able to compare the results of the 
Table 1. XMM-Newton monitoring campaign observations on IGR J16318-4848. The table shows EPIC-pn exposures times and source extraction region around the object for each observation.

\begin{tabular}{cccc}
\hline \hline \multicolumn{2}{c}{ Start time - End time (UT) } & $T_{\exp }$ & Region \\
\hline $2003-02-10 ~ 16: 58$ & $2003-02-1023: 55$ & $23000 \mathrm{~s}$ & $30^{\prime \prime}$ \\
$2004-02-1802: 43$ & $2004-02-1808: 16$ & $17872 \mathrm{~s}$ & $30^{\prime \prime}$ \\
$2004-03-20 ~ 22: 13$ & $2004-03-2104: 47$ & $20213 \mathrm{~s}$ & $22.5^{\prime \prime}$ \\
$2004-08-2004: 30$ & $2004-08-2010: 04$ & $18936 \mathrm{~s}$ & $30^{\prime \prime}$ \\
\hline
\end{tabular}

radiation transfer simulations by Matt et al. (1999) and Matt (2002) with the spectra obtained through the coordinated observations, in order to determine the time evolution of the following quantities characterizing the accretion flow: column density along the line of sight, covering fraction of the line-emitting matter, some constraints on the geometry, and ionization state of the matter.

This paper is organized as follows: in Sect. 2 information about the observations and the data reduction are given, while in Sects. 3 and 4 the results are presented and discussed.

\section{Observations and data reduction}

\subsection{XMM-Newton}

The monitoring campaign consists of three observations spaced by periods of one month and five months, respectively. In this paper, we present a detailed study of these three observations along with the reanalysis of the XMM-Newton observation performed in February 2003. Table 1 summarizes the information on all XMM-Newton observations covered by this paper, together with the EPIC-pn total exposure time and the extraction region used for each observation. The size of the extraction region for each observation was selected so as to optimise the signal to noise ratio.

We only discuss the EPIC-pn data (Strüder et al. 2001) as this instrument has the largest effective area above $5 \mathrm{keV}$. We have analysed the EPIC-mos data and found that it does not contribute significantly to the results. Also, OM data was not considered since it is outside the scope of this work.

All data sets were analyzed using SAS v.6.1.0 (Gabriel et al. 2004). Following the standard procedure for XMM-Newton data reduction, we used the most recent calibration files available in September 2004.

The spectra were accumulated using single- and doubleevents (events where the charge in the CCD is collected in either one or two adjacent pixels). Pile-up effects were checked using the epaplot task and were found to be negligible in all observations. The background contribution was always small, except in the observation of March 2004, which was affected by high background radiation. Time intervals for which background flaring does not improve the signal to noise in the EPIC-pn observations were excluded from the analysis following the method described in Piconcelli et al. (2004). Table 1 shows the exposures times after correction for background flaring activity.

All the spectra have been rebinned, in order to ensure that each background-subtracted spectral channel has at least 25 counts, and that the instrumental energy resolution is not oversampled by a factor larger than 3 .

\subsection{INTEGRAL}

In Table 2 we present the observing log of the INTEGRAL (Winkler et al. 2003) observations which were performed
Table 2. INTEGRAL monitoring campaign observations on IGR J16318-4848. The table shows the start time and end time of IBIS/ISGRI INTEGRAL observations on this source.

\begin{tabular}{cc}
\hline \hline \multicolumn{2}{c}{ Start time - End time (UT) } \\
\hline $2004-02-18$ 03:41 & 2004-02-18 17:29 \\
$2004-03-20 ~ 20: 15$ & $2004-03-2112: 35$ \\
$2004-08-19 ~ 13: 46$ & $2004-08-2022: 41$ \\
\hline
\end{tabular}

simultaneously with XMM-Newton. In the first two observations IGR J16318-4848 was the main target and the observations were done using a so-called $5 \times 5$ rectangular dither pattern. In the third observation the main target was the nearby ( $\sim 56^{\prime}$ away) source IGR J16320-4751 (Rodriguez et al. 2006) and the observation was performed using a so-called hexagonal dither pattern. The $5 \times 5$ rectangular dither pattern consists of a square pattern around the nominal target location (1 source on-axis pointing, 24 off-source pointings, with a 2 degrees step), while the hexagonal dither pattern consists of a hexagonal pattern around the nominal target location (1 source on-axis pointing, 6 off-source pointings, each 2 degrees apart). IGR J16318-4848 was always in the fully coded field of view of both the IBIS and SPI instruments, where the instrumental response is optimal.

In this paper we only consider data from IBIS/ISGRI (Lebrun et al. 2003 and Ubertini et al. 2003) and use only those data that overlap with the XMM-Newton observations. We do not consider the data from the IBIS/PICsIT, SPI, Jem-X or $\mathrm{OMC}$ instruments, since either the angular resolution is too high (SPI: $2.5^{\circ}$ ) and therefore the various sources in the Norma region of the Galaxy close to each other make a proper analysis impossible (e.g., Rodriguez et al. 2006), or IGR J16318-4848 was too weak to be detected (IBIS/PICsIT, Jem-X, OMC).

The INTEGRAL data reduction and the extraction of the events were performed using one of the latest versions of the Offline Scientific Analysis (OSA; Courvoisier et al. 2003), i.e., v5.0. The data from the IBIS/ISGRI instrument were used to produce images in the $20-40$ and $40-80 \mathrm{keV}$ energy ranges, with the aim of effectively identifying the most active sources in the field. From the results of this step, we produced a catalogue of active sources which was given as an input for a second run producing images in the 20-60 and 60-200 keV energy ranges; we increased the first range up to $60 \mathrm{keV}$ to increase the signal to noise ratio in the lower energy band. In the latter we forced the extraction/cleaning of each of the catalogue sources in order to obtain the most reliable results for IGR J16318-4848 (see Goldwurm et al. 2003 for a detailed description of the IBIS analysis software). These results were used in the production of the energy spectra for IGR J16318-4848. Note that in all these processes, we restricted the data reduction to the times that were strictly simultaneous with the XMM-Newton observations (see Table 1). To do so we created good time interval fits files that were provided as an input to OSA; we also restricted the spectral energy range to $20-60 \mathrm{keV}$, since no significant emission was detected above these energies.

\section{Results}

\subsection{Timing analysis}

In Fig. 1, we show the IGR J16318-4848 light curves in the energy ranges, 8-15 keV (upper panel) and 6-6.6 keV (middle panel), together with the ratio of the two light curves (lower panel). We used a bin time of $1024 \mathrm{~s}$, although we also 

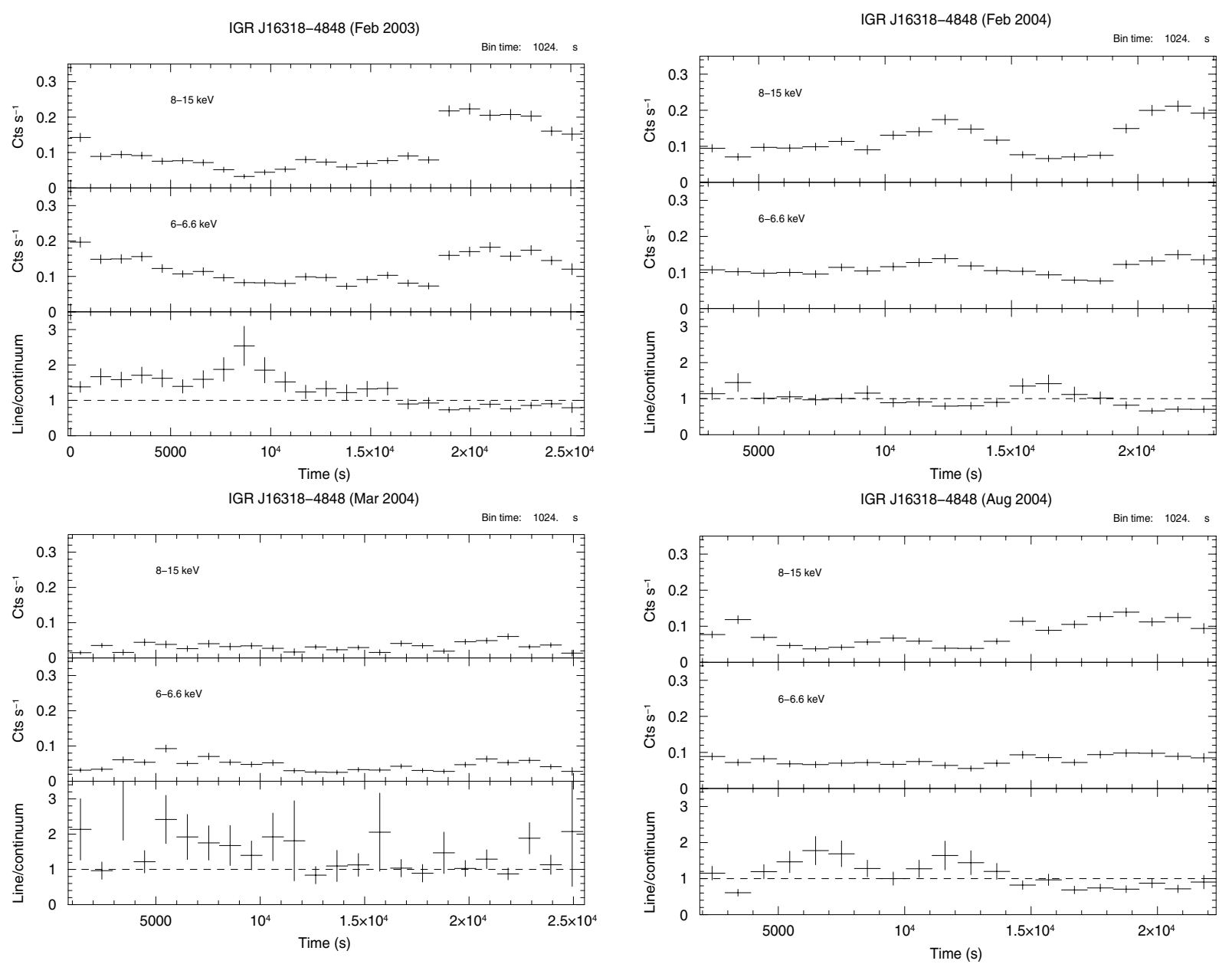

Fig. 1. XMM-Newton EPIC-pn light curves in the 8-15 keV (upper panel) and 6-6.6 keV (middle panel) energy ranges. In the lower panel, the ratio of the two light curves is shown (bin time $=1024 \mathrm{~s}$ ).

studied several other bin times, such as 256 and $512 \mathrm{~s}$, but they do not provide further improvement on the data quality. In this plot we can clearly see the February 2003 high energy flare that was studied by Matt \& Guainazzi (2003) and variations by a factor $2 \sim 3$ in $\leq 5 \times 10^{3} \mathrm{~s}$ are visible also in the February 2004 light curve, and a short flare in the August 2004 light curve.

Only the February 2003 observation shows a significant variation in the ratio of the two light curves. This effect could indicate variations of the properties of the cold matter on times scales of about $10^{4} \mathrm{~s}$ (Matt \& Guainazzi 2003). This variation in the continuum flux with respect to the flux in the $\mathrm{Fe} \mathrm{K}_{\alpha}$ line is not present in the rest of the observations.

In Fig. 2, we show the count rate in the $8-15 \mathrm{keV}$ energy range, which is dominated by the continuum, and the count rate in the $6-6.6 \mathrm{keV}$ energy range, which is dominated by the $\mathrm{Fe} \mathrm{K}_{\alpha}$ line, divided by the average count rate in each band. During the February 2003, February 2004 and August 2004 observations, a flux increment can be clearly seen. In all cases the $\mathrm{Fe} \mathrm{K}_{\alpha}$ line follows almost immediately the continuum. This suggests that the emission lines are produced by the illumination of optically-thick matter on a small scale around the high-energy continuum source. These results are consistent with Walter et al. (2003). However, the variation of the continuum exhibits a larger dynamical range than the variation of the $\mathrm{Fe} \mathrm{K}_{\alpha}$ iron line (see Fig. 3). This suggests that at least part of the line emission comes from regions, that do not respond on small timescales to the continuum.

\subsection{Spectral analysis}

In Fig. 4 all four IGR J16318-4848 spectra are shown. Despite the large flux variability, the spectral shapes are very similar to one another. The spectral analysis has been performed using events above $5 \mathrm{keV}$ because the source is highly absorbed below this energy. Detections at a level greater than $3 \sigma$ have been found only in the February 2004 and August 2004 observations (in Table 3 we shown the count rate (CR) in the $1-5 \mathrm{keV}$ energy band).

Following Matt \& Guainazzi (2003), the spectra of all available XMM-Newton observations of IGR J16318-4848 have been fitted (using XSPEC v.11.3.1), alongside the simultaneous INTEGRAL spectra whenever available, with the following baseline model:

$F(E)=\mathrm{e}^{-N_{\mathrm{H}} \sigma(E)}\left[A E^{-\Gamma} \mathrm{e}^{E / E_{\mathrm{c}}}+\sum_{i=1}^{4} G_{i}(E)+\sum_{i=1}^{2} S_{\mathrm{cs}_{i}}(E)\right]$

where $\sigma(E)$ is the sum of the photoelectric and the Thompson cross-sections, $G_{i}(E)$ are Gaussian profiles corresponding to the $\mathrm{Fe} \mathrm{K}_{\alpha}$ doublet, $\mathrm{Fe} \mathrm{K}_{\beta}$ and $\mathrm{Ni} \mathrm{K}_{\alpha}$ fluorescent emission lines, and $S_{\mathrm{cs}_{i}}(E)$ is the $\mathrm{Fe}_{\alpha 1}$ and $\mathrm{Fe}_{\alpha 2}$ Compton Shoulder respectively. The energy first component of the Fe $\mathrm{K}_{\alpha}$ doublet has been left free to vary in the fit, while the energy centroid of the second component of the iron line doublet was fixed to the energy of the first component multiplied by 1.002 (Kranse et al. 1979), as for neutral iron. The relative intensity of the second component 

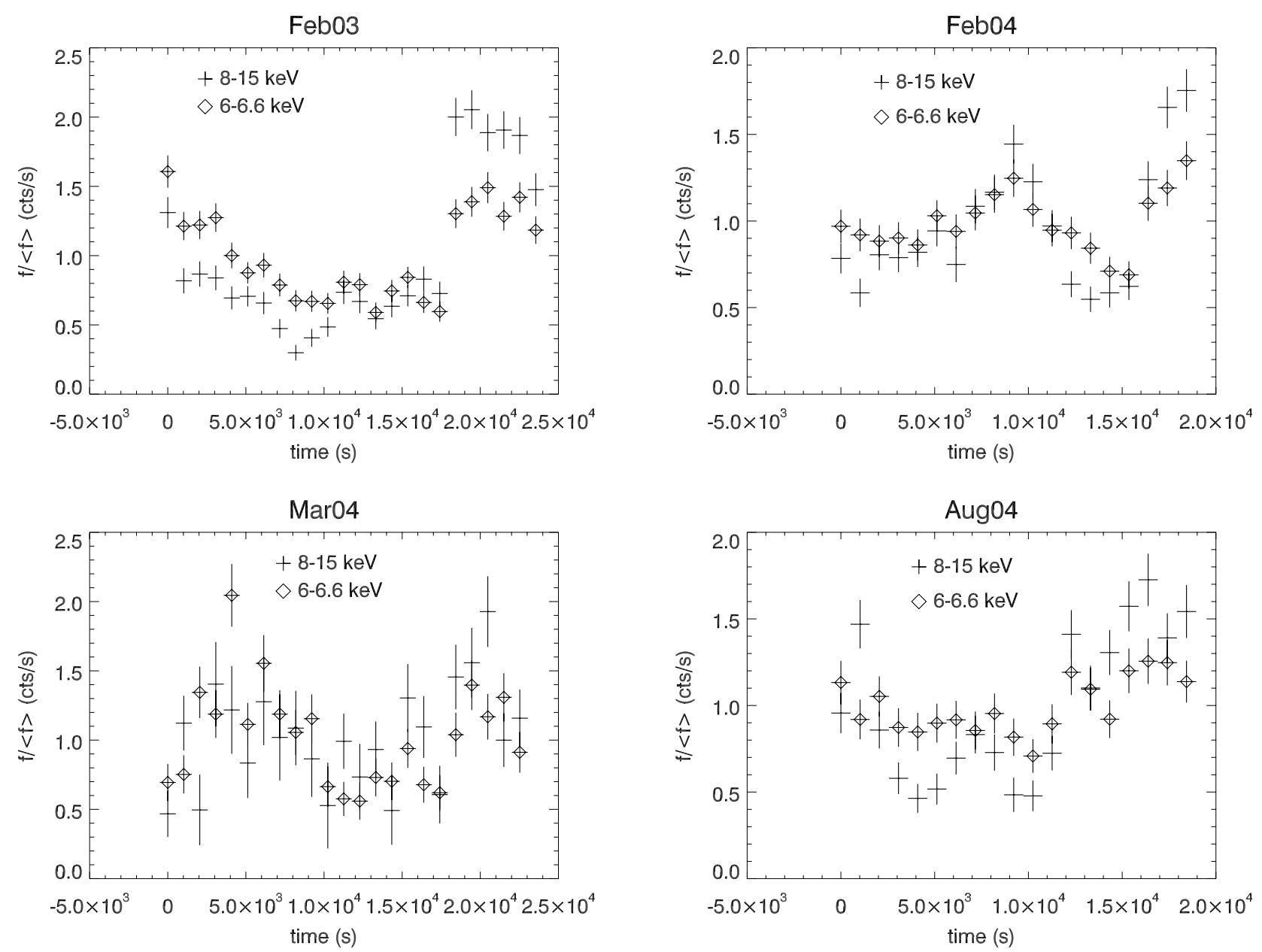

Fig. 2. XMM-Newton count rate divided by the average count rate in each band. The rapid flux increments in the February 2003, February 2004 and August 2004 observations take places simultaneously in both bands.

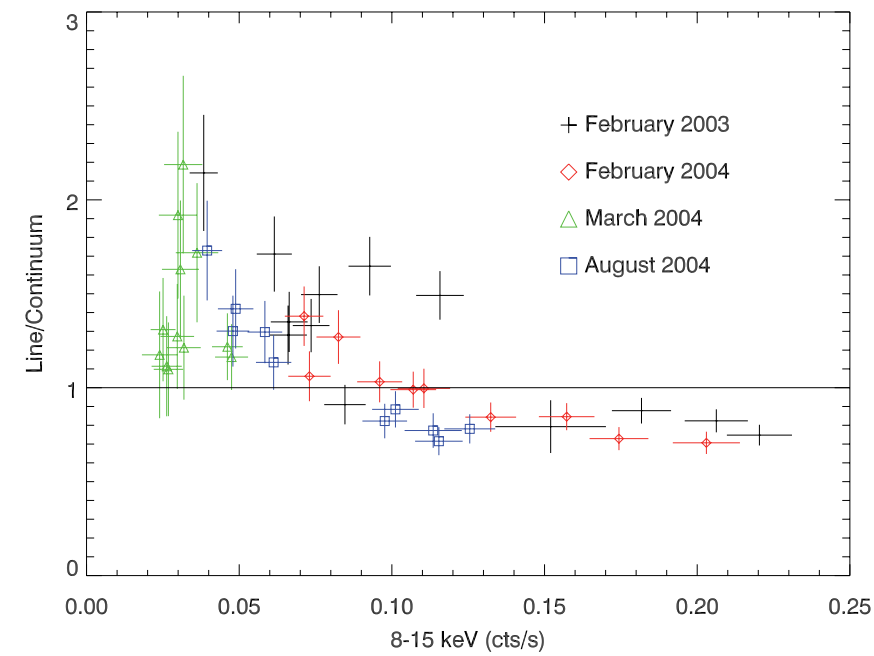

Fig. 3. Ratio between the $6-6.6 \mathrm{keV}$ and the $8-15 \mathrm{keV}$ energy band as a function of the $8-15 \mathrm{keV}$ count rate.

of the iron doublet was fixed to twice the intensity of the first component, as dictated by atomic physics.

The last component of the baseline model was modeled using two box functions (as a first order approximation) corresponding to the Compton Shoulder (CS) of Fe $\mathrm{K}_{\alpha}$ doublet (e.g. Sunyaev \& Churazov 1996; Matt 2002; Watanabe et al. 2003).

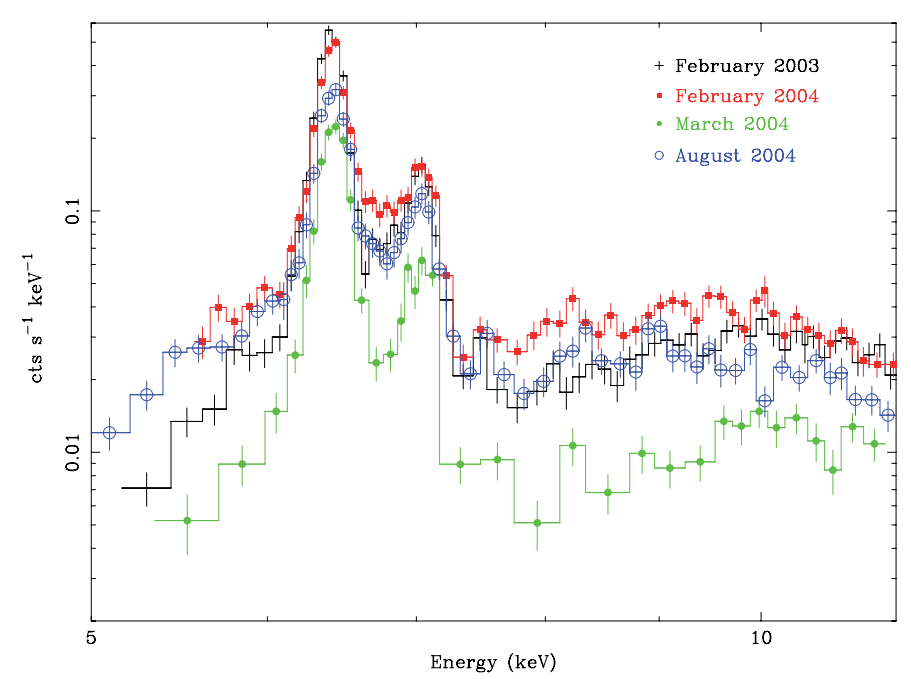

Fig. 4. Spectra of the four IGR J16318-4848 XMM-Newton observations. Despite the large flux variations between different observations, the spectral shapes follow the same trend.

The first box function corresponding to the lowest energy component of the iron doublet was extended in the closed interval $\left[E_{1}-0.16 \mathrm{keV}, E_{1}-0.01 \mathrm{keV}\right]$ as appropriate for the onescattering CS (Matt 2002), where $E_{1}$ is the best-fit centroid energy for the $\mathrm{Fe} \mathrm{K}_{\alpha 1}$ line, and $0.16 \mathrm{keV}$ is the maximum recoil 
energy. We neglected the higher order shoulders, i.e. the ones dominated by two or more scatterings, because they are much fainter even for Compton-thick media, both in the reflection (Matt et al. 1991; George \& Fabian 1991) and in the transmission (Leahy \& Creighton 1993) cases. Similarly, the box model for the second component was extended in the closed interval [ $\left.E_{2}-0.16 \mathrm{keV}, E_{2}-0.01 \mathrm{keV}\right]$.

In principle, one should include in the fit the Compton Shoulder for the other fluorescent lines as well. However, these lines are much fainter, and their Compton Shoulder are almost undetectable in the EPIC-pn spectra. Therefore, for simplicity they will not be considered in what follows. For the same reason, the $\mathrm{Fe} \mathrm{K}_{\beta}$ and $\mathrm{Ni} \mathrm{K}_{\alpha}$ lines were modeled as single Gaussians.

Regarding the absorbing matter, the abundance (normalized to the solar value) of elements with $Z \geq 26,\left(Z_{\mathrm{Fe}}\right.$ hereafter) was allowed to vary independently of the abundance of the lighter elements. The relative iron abundance values (Anders \& Grevesse 1989) obtained for each observation were: $0.81 \pm 0.03,0.77 \pm_{0.04}^{0.03}$, $0.89 \pm_{0.06}^{0.05}$ and $0.86 \pm_{0.06}^{0.05}$ for the February 2003, February 2004, March 2004 and August 2004 observations, respectively. These values are compatible within the errors and therefore we calculated the weighted mean, which was $0.81 \pm 0.02$, and used it in all subsequent spectral fits.

XMM-Newton and INTEGRAL spectra were fitted simultaneously, applying the relative normalization between the EPICpn and ISGRI data given by Kirsch et al. (2005).

We studied the width of the emission lines in order to avoid any possible numerical problem related to unresolved lines. Firstly, we fit all the spectra with the line width fixed to $10^{-4} \mathrm{eV}$. We then allowed the line width of the emission lines to vary, but no improvements in the $\chi^{2}$ was found. With these conditions, we obtained very tight upper limits for the width of the emission lines, smaller than $10 \mathrm{eV}$. The only exception is the August 2004 observation, when the width is mostly unconstrained. We then fixed the line width to $10^{-4} \mathrm{eV}$ for all the observations.

Despite the strong coupling between $\Gamma$ and $N_{\mathrm{H}}$ that was found in the February 2003 observations (Matt \& Guainazzi 2003), we have been able to find a stable spectral index for all the observations, with the exception of August 2004 (see Fig. 5). This has been posible due to the wide energy coverage introduced by the simultaneous INTEGRAL data, the better constraint on the metal abundance and the inclusion in our model of the Fe $\mathrm{K}_{\alpha}$ doublet (see Fig. 5).

In Table 3 we summarize the best-fit parameters of the baseline model and in Fig. 6 we present the spectra and residuals of the data against the baseline model. All the errors shown in this paper correspond to a $90 \%$ confidence level for one interesting parameter. The values of the equivalent widths in the table were computed with respect to the unabsorbed continuum.

In all 2004 observations, the relative intensity of the Compton Shoulder is higher than in the 2003 observation. Interestingly, for these observations the centroid of the $\mathrm{Fe} \mathrm{K}_{\alpha 1}$ line is significantly bluer than expected for neutral iron $(6.40 \mathrm{keV})$. This seems to suggest significant ionization of iron (Fe $\geq$ XIV, Kallman et al. 2004). However, the strong iron $\mathrm{Fe} \mathrm{K}_{\beta}$ line implies that iron is less ionized than Fe XI or so (Molendi et al. 2003). The apparent blueshift may then be an instrumental effect, or an artifact due to either the oversimplified $\mathrm{CS}$ modeling or to the assumption of a narrow line core. We recall that the systematic uncertainties on the gain of the EPIC-pn camera at $6 \mathrm{keV}$ is $10 \mathrm{eV}$ (Kirsch 2006).

In Fig. 7 we show the residuals of the Compton-Shoulder feature for all the observations. We have also included in Table 3 the $\chi^{2}$ values of our baseline model without the Compton

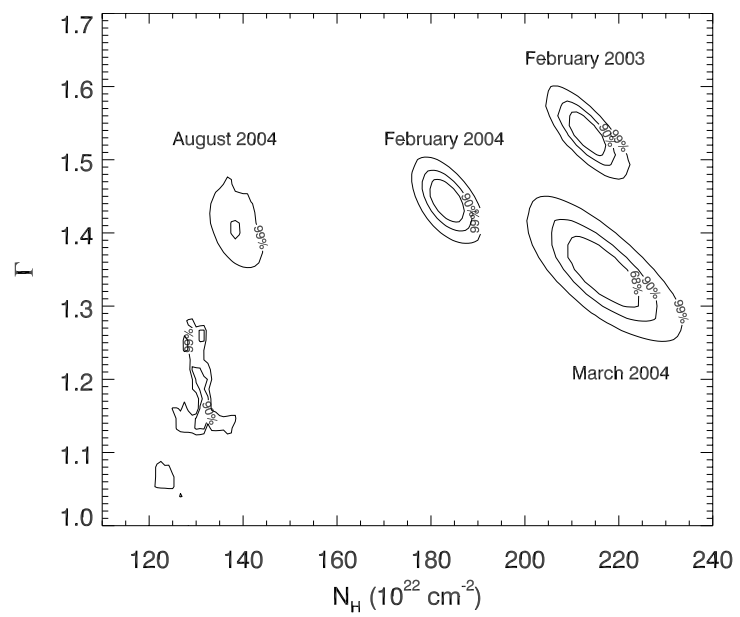

Fig. 5. $\Gamma-N_{\mathrm{H}}$ ISO- $\chi^{2}$ contour plots corresponding to IGR J16318-4848 XMM-Newton observations.

Shoulder. In all the observations the improvement when adding the CS is statistically significant.

A comparison between the ratio of all INTEGRAL spectra and their mean spectrum was performed, showing no trend. This stable spectral shape agrees with the accreting pulsar objects.

Interestingly, Matt \& Guainazzi (2003) report soft excess emission above the extrapolation of the baseline model at energies $E \lesssim 5 \mathrm{keV}$. We have reanalyzed the images obtained during the monitoring campaign and discovered a serendipitous source at $\simeq 30^{\prime \prime}$ from the IGR J16318-4848 centroid position. This serendipitous source is responsible for the reported soft excess. In the other three observations, this soft source falls into the CCD gaps. Still, we detect soft X-ray emission at a significance level of more than $3 \sigma$ in two out of the four observations. No spectral information can be derived on this component from the available data.

\section{Discussion}

We have presented results from a coordinated XMMNewton/INTEGRAL monitoring campaign on IGR J163184848. The source was always clearly detected, albeit with a large amplitude (a factor of 3 ) flux variations, suggesting (along with its rediscovery in the ASCA archive) that it is a persistent source that escaped attention before 2003 only due to the strong absorption. Persistence of the X-ray emission is a characteristic of Be systems with wide orbits (hundreds of days, Negueruela 1998).

The properties of the circumstellar matter clearly changed from one observation to the other, the column density (as derived from absorption) for instance being significantly lower (but still Compton-thick) in the last observation. As a further probe of the circumstellar matter, we use the Compton Shoulder, which depends on the covering fraction of the matter and on its average column density (Matt 2002; Matt \& Guainazzi 2003), while the absorption gives information on the line-of-sight (l.o.s.) column only.

In Fig. 8 we show the results of the $\mathrm{Fe}_{\alpha}$ Compton Shoulder normalized intensity, $f_{\mathrm{CS}}$ (the ratio between the CS and the line core intensities) against the $\mathrm{EW}$ of the $\mathrm{Fe} \mathrm{K}_{\alpha}$. Comparing our results with the predictions of radiative transfer simulations in Matt (2002), a covering fraction of the obscuring matter in the range $0.1-0.3$, and average column densities in the range $2 \times 10^{22}$ to $5 \times 10^{23} \mathrm{~cm}^{-2}$ are derived. 
Table 3. Best-fit parameters and results of the IGR J16318-4848 monitoring campaign using the baseline model.

\begin{tabular}{|c|c|c|c|c|}
\hline Parameter & February 2003 & February 2004 & March 2004 & August 2004 \\
\hline$\Gamma$ & $1.54_{-04}^{+0.02}$ & $1.46 \pm 0.03$ & $1.35 \pm 0.04$ & $1.41 \pm 0.03$ \\
\hline$E_{\mathrm{c}}[\mathrm{keV}]$ & $58_{-11}^{+30}$ & $<16$ & $<180$ & $<30$ \\
\hline $\mathrm{A}\left[10^{-2} \mathrm{~cm}^{-2} \mathrm{~s}^{-1}\right]$ & $35 \pm 1$ & $27.0_{-0.7}^{+0.8}$ & $9.95 \pm 0.01$ & $4.8_{-0.2}^{+0.1}$ \\
\hline $\mathrm{HR}\left[\frac{20-30}{30-50}\right][\mathrm{keV}]$ & $\ldots$ & $0.40 \pm 0.14$ & $0.46 \pm 0.04$ & $0.44 \pm 0.10$ \\
\hline$N_{\mathrm{H}}\left[10^{24} \mathrm{~cm}^{-2}\right]$ & $2.12 \pm 0.03$ & $1.82_{-0.03}^{+0.05}$ & $2.16_{-0.06}^{+0.07}$ & $1.38_{-0.03}^{+0.04}$ \\
\hline$E_{1}\left(\mathrm{Fe} \mathrm{K} \mathrm{K}_{\alpha 1}\right)[\mathrm{keV}]$ & $6.39 \pm 0.01$ & $6.43_{-0.02}^{+0.01}$ & $6.44 \pm 0.01$ & $6.42_{-0.04}^{+0.05}$ \\
\hline$I_{1}\left(\mathrm{Fe} \mathrm{K} \mathrm{K}_{\alpha}\right)\left[10^{-4} \mathrm{~cm}^{-2} \mathrm{~s}^{-1}\right]$ & $1.71 \pm 0.02$ & $0.93 \pm 0.04$ & $0.83_{-0.02}^{+0.04}$ & $0.65_{-0.03}^{+0.06}$ \\
\hline$E W\left(\mathrm{Fe} \mathrm{K}_{\alpha}\right)[\mathrm{eV}]$ & $9.5 \pm 0.3$ & $8.0 \pm 0.4$ & $8.6_{-0.5}^{+0.0}$ & $23.9_{-1.1}^{+2.1}$ \\
\hline$E_{2}\left(\mathrm{Fe} \mathrm{K} \mathrm{K}_{\beta}\right)[\mathrm{keV}]$ & $7.05 \pm 0.01$ & $7.10_{-0.02}^{+0.03}$ & $7.05_{-0.01}^{+0.02}$ & $7.05_{-0.02}^{+0.04}$ \\
\hline$I_{2}{ }^{a}$ & $0.20 \pm 0.02$ & $0.24_{-0.06}^{+0.03}$ & $0.18_{-0.05}^{+0.03}$ & $0.25 \pm 0.05$ \\
\hline$E W\left(\mathrm{Fe} \mathrm{K}_{\beta}\right)[\mathrm{eV}]$ & $2.2_{-0.2}^{+0.3}$ & $2.3_{-0.5}^{+0.3}$ & $2.8_{-0.7}^{+0.3}$ & $7.1 \pm 1.4$ \\
\hline$E_{3}\left(\mathrm{Ni} \mathrm{K}_{\alpha}\right)[\mathrm{keV}]$ & $7.45_{-0.01}^{+0.06}$ & $7.45_{-0.01}^{+0.1}$ & $7.5_{-0.1}^{+0.4}$ & $7.5_{-0.7}^{+0.4}$ \\
\hline$I_{3}{ }^{a}$ & $0.05_{-0.01}^{+0.01}$ & $0.05_{-0.02}^{+0.01}$ & $0.03_{-0.01}^{+0.02}$ & $0.07_{-0.05}^{+0.03}$ \\
\hline$E W\left(\mathrm{Ni} \mathrm{K}_{\alpha}\right)[\mathrm{eV}]$ & $0.7_{-0.1}^{+0.2}$ & $0.5_{-0.2}^{+0.4}$ & $0.5_{-0.3}^{+0.4}$ & $2.2_{-1.3}^{+0.8}$ \\
\hline$I_{\mathrm{CS}}{ }^{a}$ & $0.05 \pm 0.03$ & $0.25 \pm 0.06$ & $0.24_{-0.06}^{+0.13}$ & $0.19_{-0.07}^{+0.08}$ \\
\hline$\chi^{2} / v$ & $106.9 / 92$ & 98.9/97 & $64.9 / 51$ & $111.7 / 83$ \\
\hline$\chi^{2} / v($ Without CS $)$ & $120.9 / 93$ & $153.0 / 98$ & $114.85 / 52$ & $135.85 / 84$ \\
\hline$F\left[10^{-11} \mathrm{erg} \mathrm{cm}^{-2} \mathrm{~s}^{-1}\right]$ & $2.39_{-0.06}^{+0.03}$ & $2.11 \pm 0.08$ & $0.8_{-0.2}^{+0.5}$ & $1.2_{-0.2}^{+0.3}$ \\
\hline$C R(1-5 \mathrm{keV})\left[10^{-3} \mathrm{cts} \mathrm{s}^{-1}\right]$ & $1.3 \pm 0.6$ & $12.9 \pm 1.4$ & $<2.5$ & $2.10 \pm 0.40$ \\
\hline
\end{tabular}

${ }^{a}$ Ratio intensity against the $\mathrm{Fe} \mathrm{K}_{\alpha}$.
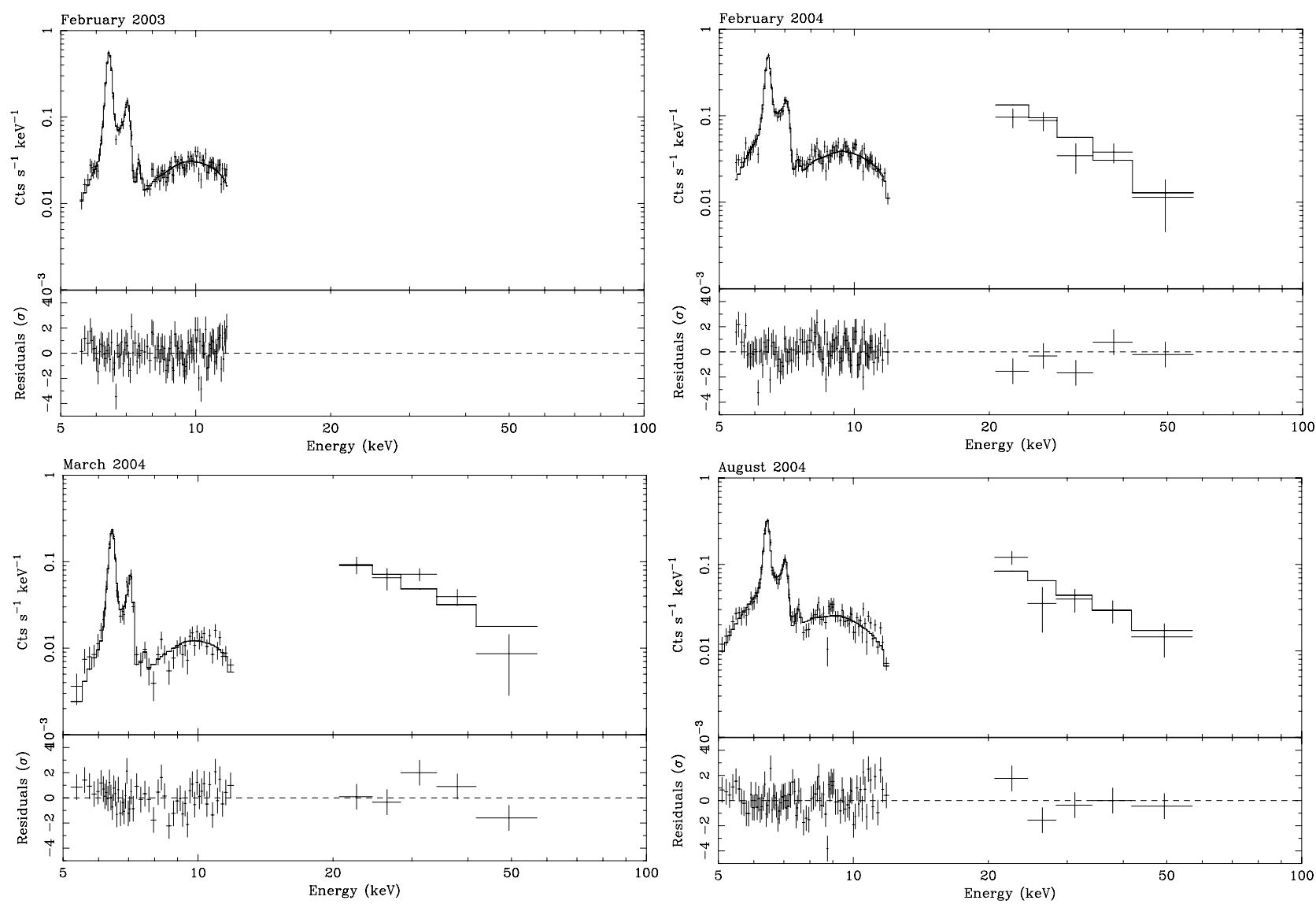

Fig. 6. Spectra (upper panels) and residuals in units of standard deviations (lower panels) when the baseline model is applied to the IGR J16138-4848 observations discussed in this paper. 


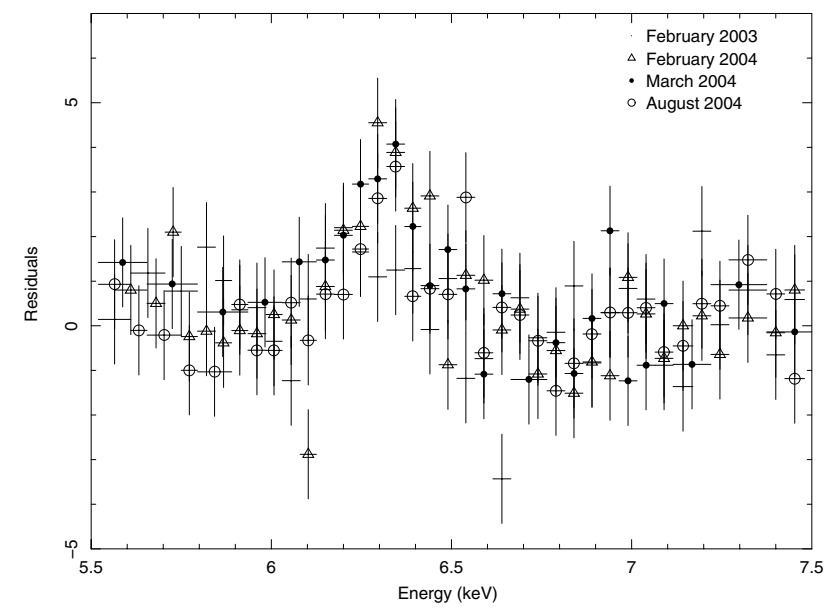

Fig. 7. Residuals of the Compton-Shoulder feature for all of the IGR J16318-4848 observations.

The average column density is always much lower ( 1 to 2 orders of magnitude) than the 1.o.s. one. The fact that the two values are very different implies that the circumstellar matter is highly inhomogeneous. As the average value is systematically smaller than that of the 1.o.s. this strongly suggests that we are observing the source from a privileged direction. The relatively small covering factor rules out the hypothesis that the compact object (a neutron star according to Filliatre \& Chaty 2004, cf., Negeruela 1998) is embedded in the wind from the giant companion (a sgB[e] star according to Filliatre \& Chaty 2004). This is not surprising if the system indeed has a wide orbit, as suggested by its persistence, this can be interpreted in terms of a flattened distribution of matter (quite plausible in a binary system, especially if it has a wide orbit), with the column density decreasing from the equatorial plane, and the line of sight lying close to that plane. Even if this scenario is not unique, it is plausible. The variations in the covering factor and average column density may then indicate variations in the mass transfer rate, possibly due to a highly eccentric orbit of the system.

Acknowledgements. This paper is based on observations obtained with XMMNewton, an ESA science mission with instruments and contributions directly funded by ESA Member States and the USA (NASA).

\section{References}

Anders, E., \& Grevesse, N. 1989, Geochim. Cosmochim. Acta, 53, 197

Courvoisier, T. J.-L., Walter, R., Rodriguez, J., Bouchet, L., \& Loutovinon, A. A. 2003, IAUC, 8063

de Plaa, J., et al. 2003, Astronomer's Telegram, 119

Filliatre, P., \& Chaty, S. 2004, ApJ, 616, 469

Gabriel, C., Denby, M., Fyfe, D. J., et al. 2004, in ADASS XIII, ed. F. Ochsenbein, M. Allen, \& D. Egret (San Francisco: ASP), ASP Conf. Ser., 314,759

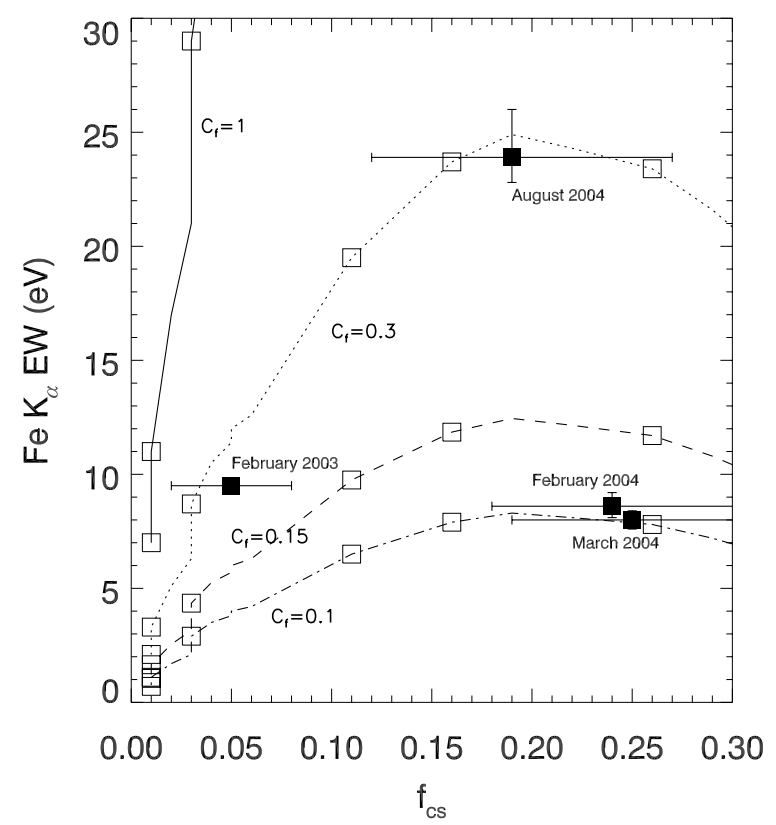

Fig. 8. Fe $\mathrm{K}_{\alpha}$ fluorescent line EW against the Compton Shoulder normalized intensity $\left(f_{\mathrm{CS}}\right)$. The lines represent the predictions of radiative transfer simulations after Matt (2002) for different values of the gas covering fraction, $C_{f}$. The filled squares represent the four XMM-Newton measurements discussed in this paper. The empty squares indicate the position along the lines corresponding to the following values of column density of the obscuring gas: $N_{\mathrm{H}}=10^{22}, 2 \times 10^{22}, 5 \times 10^{22}, 10^{23}$, $2 \times 10^{23}$, and $5 \times 10^{23} \mathrm{~cm}^{-2}$.

George, I. M., \& Fabian, A. C. 1991, MNRAS, 249, 352

Goldwurm, A., David, P., Foschini, L., et al. 2003, A\&A, 411, L223

Hynes, R. I., Clark, J. S., Barsukova, E. A., et al. 2002, A\&A, 392, 991

Kallman, G., et al. 2004, COSPAR Scientific Assembly, Paris, 3872

Kirsch, M. G. F., et al. 2005 [arXiv: astro-ph/0508235]

Kirsch, M. G. F. 2006, XMM-SOC-CAL-TN-0018

Kranse, M. O., \& Oliver J. H. 1979, J. Phys. Chem. Ref. Data, 8, 239

Kuulkers, E. 2005, AIPC, 797, 402

Leahy, D. A., \& Creighton, J. 1991, MNRAS, 263, 314

Lebrun, F., Leray, J. P., Lavocat, P., et al. 2003, A\&A, 411, L141

Matt, G. 2002, MNRAS, 337, 147

Matt, G., \& Guainazzi, M. 2003, MNRAS, 341, L13

Matt, G., Perola, G. C., \& Piro, L. 1991, A\&A, 247, 25

Molendi, S., Bianchi, S., \& Matt, G. 2003, MNRAS, 343, L1

Murakami, H., Dotani, T., \& Wijnands, R. 2003, IAUC, 8070

Negueruela, I. 1998, A\&A, 338, 505

Piconcelli, E., Jimenez-Bailón, E., Guainazzi, M., et al. 2004, MNRAS, 351, 161

Revnivtsev, M., Sazonov, S., Gifanov, M., \& Sunyaev, R. 2003, Astron. Lett., 29, 587

Rodriguez, J., Bodaghee, A., Kaaret, P., et al. 2006, MNRAS, 366, 274

Schartel, N., Ehle, M., Breitfellner, M., et al. 2003, IAUC, 8072

Strüder, L., Briel, U., Dennerl, K., et al. 2001, A\&A, 365, L18

Sunyaev, R. A., \& Churazov, E. M. 1996, Astron. Lett., 22, 648

Ubertini, P., Lebrun, F., Di Cocco, G., et al. 2003, A\&A, 411, L131

Walter, R., Rodriguez, J., Foschini, L., et al. 2003, A\&A, 411, L427

Watanabe, S., Sako, M., Ishida, M., et al. 2003, ApJ, 597, L37

Winkler, C., Courvoisier, T. J.-L., Di Cocco, G., et al. 2003, A\&A, 411, L1 